\title{
Front Matter: Volume 7653
}

, "Front Matter: Volume 7653," Proc. SPIE 7653, Fourth European Workshop on Optical Fibre Sensors, 765301 (9 September 2010); doi:

$10.1117 / 12.868580$

EviE Event: (EWOFS'10) Fourth European Workshop on Optical Fibre Sensors, SPIE. 2010, Porto, Portugal 


\title{
PROCEEDINGS OF SPIE
}

\section{Fourth European Workshop on Optical Fibre Sensors}

\author{
José Luís Santos \\ Brian Culshaw \\ José Miguel López-Higuera \\ William N. MacPherson \\ Editors \\ 8-10 September 2010 \\ Porto, Portugal \\ Organized by \\ INESC Porto (Portugal) \\ University of Porto (Portugal) \\ Cooperating Organizations \\ SPIE \\ OSA-The Optical Society \\ IEEE Photonics Society \\ EOS-European Optical Society \\ SPF-Portuguese Physical Society \\ Published by \\ SPIE
}

Volume 7653 
The papers included in this volume were part of the technical conference cited on the cover and title page. Papers were selected and subject to review by the editors and conference program committee. Some conference presentations may not be available for publication. The papers published in these proceedings reflect the work and thoughts of the authors and are published herein as submitted. The publisher is not responsible for the validity of the information or for any outcomes resulting from reliance thereon.

Please use the following format to cite material from this book:

Author(s), "Title of Paper," in Fourth European Workshop on Optical Fibre Sensors, edited by José Luís Santos, Brian Culshaw, José Miguel López-Higuera, William N. MacPherson, Proceedings of SPIE Vol. 7653 (SPIE, Bellingham, WA, 2010) Article CID Number.

ISSN 0277-786X

ISBN 9780819480835

Published by

SPIE

P.O. Box 10, Bellingham, Washington 98227-0010 USA

Telephone +1 3606763290 (Pacific Time) · Fax +1 3606471445

SPIE.org

Copyright (c) 2010, Society of Photo-Optical Instrumentation Engineers

Copying of material in this book for internal or personal use, or for the internal or personal use of specific clients, beyond the fair use provisions granted by the U.S. Copyright Law is authorized by SPIE subject to payment of copying fees. The Transactional Reporting Service base fee for this volume is $\$ 18.00$ per article (or portion thereof), which should be paid directly to the Copyright Clearance Center (CCC), 222 Rosewood Drive, Danvers, MA 01923. Payment may also be made electronically through CCC Online at copyright.com. Other copying for republication, resale, advertising or promotion, or any form of systematic or multiple reproduction of any material in this book is prohibited except with permission in writing from the publisher. The CCC fee code is 0277-786X/10/\$18.00.

Printed in the United States of America.

Publication of record for individual papers is online in the SPIE Digital Library.

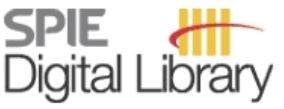

SPIEDigitalLibrary.org

Paper Numbering: Proceedings of SPIE follow an e-First publication model, with papers published first online and then in print and on CD-ROM. Papers are published as they are submitted and meet publication criteria. A unique, consistent, permanent citation identifier (CID) number is assigned to each article at the time of the first publication. Utilization of CIDs allows articles to be fully citable as soon they are published online, and connects the same identifier to all online, print, and electronic versions of the publication. SPIE uses a six-digit CID article numbering system in which:

- The first four digits correspond to the SPIE volume number.

- The last two digits indicate publication order within the volume using a Base 36 numbering system employing both numerals and letters. These two-number sets start with 00, 01, 02, 03, 04 , $05,06,07,08,09,0 A, 0 B \ldots 0 Z$, followed by 10-1Z, 20-2Z, etc.

The CID number appears on each page of the manuscript. The complete citation is used on the first page, and an abbreviated version on subsequent pages. Numbers in the index correspond to the last two digits of the six-digit CID number. 


\title{
Contents
}

\author{
xix Conference Committee \\ xxi Introduction \\ xxiii Conference Sponsors
}

SPECIAL SESSION: OPTICAL FIBRE SENSORS FOR SUSTAINABLE ENVIRONMENT

765302 A paradigm change in energy: the new electricity age (Invited Paper) [7653-160]

T. Bosselmann, Siemens AG (Germany)

765303 Temperature monitoring of geothermal energy wells (Invited Paper) [7653-166]

M. Jaaskelainen, SensorTran (United States)

\section{EWOFS2010 INTRODUCTORY OVERVIEW}

765304 Fibre optic sensor technology: An engineering reality or a scientific opportunity? (Invited Paper) [7653-162]

B. Culshaw, Univ. of Strathclyde (United Kingdom)

\section{INVITED TALKS}

765305 Multimaterial fiber sensors (Invited Paper) [7653-167]

F. Sorin, Y. Fink, Massachusetts Institute of Technology (United States)

765306 Spintronic microfluidic platform for biomedical and environmental applications (Invited Paper) [7653-169]

F. A. Cardoso, INESC MN and IN (Portugal) and Instituto Superior Técnico (Portugal);

V. C. Martins, INESC MN and IN (Portugal) and Institute of Biotechnology and Bioengineering (Portugal); L. P. Fonseca, Institute of Biotechnology and Bioengineering (Portugal);

J. Germano, L. A. Sousa, M. S. Piedade, Instituto Superior Técnico (Portugal) and INESC ID

(Portugal); P. P. Freitas, INESC MN and IN (Portugal) and Instituto Superior Técnico (Portugal)

765307 Emerging fiber optic endomicroscopy technologies towards noninvasive real-time visualization of histology in situ (Invited Paper) [7653-161]

J. Xi, Y. Zhang, L. Huo, Y. Chen, T. Jabbour, The Johns Hopkins Univ. (United States); M.-J. Li, Corning Inc. (United States); X. Li, The Johns Hopkins Univ. (United States)

765308 Fibre optic grating sensors for biofuels (Invited Paper) [7653-62]

M. Muller, J. L. Fabris, H. J. Kalinowski, Univ. Tecnológica Federal do Paraná (Brazil) 
765309 Recent progress towards centimetric spatial resolution in distributed fibre sensing (Invited Paper) [7653-170]

L. Thévenaz, S. Foaleng-Mafang, K.-Y. Song, S. Chin, J.-C. Beugnot, N. Primerov, M. Tur, Ecole Polytechnique Fédérale de Lausanne (Switzerland)

7653 OA Distributed fiber optic sensors embedded in technical textiles for structural health monitoring (Invited Paper) [7653-164]

K. Krebber, P. Lenke, S. Liehr, N. Noether, M. Wendt, A. Wosniok, Bundesanstalt für Materialforschung und -prüfung (Germany)

Wednesday, 8 September 2010

SESSION 1 PHYSICAL AND MECHANICAL SENSORS

7653 OB Fibre optic hot-wire flowmeter based on a metallic coated hybrid LPG-FBG structure [7653-132]

P. Caldas, INESC Porto (Portugal), Univ. do Porto (Portugal), and Escola Superior de Tecnologia e Gestão (Portugal); P. A. S. Jorge, INESC Porto (Portugal); G. Rego, INESC Porto (Portugal) and Escola Superior de Tecnologia e Gestão (Portugal); O. Frazão, INESC Porto (Portugal); J. L. Santos, INESC Porto (Portugal) and Univ. do Porto (Portugal); L. A. Ferreira, F. Araújo, INESC Porto (Portugal)

7653 OC Drift in high-temperature FBG sensors [7653-09]

A. Busboom, GE Global Research (Germany); H. Xia, B. K. Lee, G. P. Koste, GE Global Research (United States)

7653 OD Polarimetric sensitivity to hydrostatic pressure and temperature in birefringent dual-core microstructured polymer fiber [7653-34]

M. K. Szczurowski, T. Martynkien, G. Statkiewicz-Barabach, W. Urbanczyk, Wroclaw Univ. of Technology (Poland); D. J. Webb, Aston Univ. (United Kingdom)

7653 OE Wavelength encoded fiber sensor for extreme temperature range [7653-107]

D. Barrera, Univ. Politécnica de Valencia (Spain); V. Finazzi, G. Coviello, Institut de Ciencies Fotoniques (Spain); A. Bueno, S. Sales, Univ. Politécnica de Valencia (Spain); V. Pruneri, Institut de Ciencies Fotoniques (Spain) and Institució Catalana de Recerca i Estudis Avançats (Spain)

7653 OF Strain calibration of optical FBG-based strain sensors [7653-110]

J. Roths, A. Wilfert, P. Kratzer, F. Jülich, R. Kuttler, Munich Univ. of Applied Sciences (Germany)

7653 OG Investigation of long term stability of arc-induced gratings heat treated at high temperatures [7653-85]

G. Rego, Escola Superior de Tecnologia e Gestão (Portugal) and INESC Porto (Portugal); P. Caldas, Escola Superior de Tecnologia e Gestão (Portugal), INESC Porto (Portugal), and Univ. do Porto (Portugal); O. Ivanov, Institute of Radio Engineering and Electronics (Russian Federation) and Ulyanovsk State Univ. (Russian Federation); J. L. Santos, INESC Porto (Portugal) and Univ. do Porto (Portugal) 
$7653 \mathrm{OH} \quad$ Temperature independent bend measurement using a pi-phase shifted FBG at twice the Bragg wavelength [7653-93]

H. K. Bal, F. Sidiroglou, Z. Brodzeli, Victoria Univ. (Australia); S. A. Wade, Swinburne Univ. of Technology (Australia); G. W. Baxter, S. F. Collins, Victoria Univ. (Australia)

$7653 \mathrm{Ol}$ Investigation of sensing properties of long period gratings based on microstructured polymer optical fibres [7653-112]

J. Witt, M. Steffen, M. Schukar, K. Krebber, Bundesanstalt für Materialforschung und -prüfung (Germany)

7653 OJ Optical inclinometer based on fibre-taper-modal Michelson interferometer [7653-43] L. M. N. Amaral, O. Frazão, INESC Porto (Portugal); J. L. Santos, INESC Porto (Portugal) and Univ. do Porto (Portugal); A. B. Lobo Ribeiro, Univ. Fernando Pessoa (Portugal)

7653 OK Photonic crystal fiber sensor for magnetic field detection [7653-139]

S. M. M. Quintero, Pontifícia Univ. Católica do Rio de Janeiro (Brazil); C. Martelli, Univ. Tecnológica Federal do Paraná (Brazil); C. C. Kato, L. C. G. Valente, A. M. B. Braga,

Pontifícia Univ. Católica do Rio de Janeiro (Brazil)

$7653 \mathrm{OL} \quad$ Optimized conditions for gas light interaction in photonic crystal fibres [7653-134] I. Dicaire, J.-C. Beugnot, L. Thévenaz, Ecole Polytechnique Fédérale de Lausanne (Switzerland)

7653 OM Fibre loop mirror using a small core microstructured fibre for the simultaneous measurement of strain and temperature [7653-01]

R. M. André, M. B. Marques, INESC Porto (Portugal) and Univ. do Porto (Portugal); P. Roy, XLIM, CNRS, Univ. de Limoges (France); O. Frazão, INESC Porto (Portugal)

$7653 \mathrm{ON} \quad \mathrm{H}_{2}$ sensing performance of optical fibers coated with $\mathrm{WO}_{3}$ film [7653-103] J. Ou, RMIT Univ. (Australia); M. H. Yaacob, RMIT Univ. (Australia) and Univ. Putra Malaysia (Malaysia); M. Breedon, K. Kalantar-Zadeh, W. Wlodarski, RMIT Univ. (Australia)

765300 Optical refractometer based on high birefringence Bragg grating Fabry-Perot cavity [7653-151]

C. Jesus, INESC Porto (Portugal) and Univ. da Madeira (Portugal); O. Frazão, P. A. S. Jorge, INESC Porto (Portugal); J. M. Baptista, Univ. da Madeira (Portugal) and INESC Porto (Portugal)

7653 OP Transverse strain sensor based on etched phase-shifted fiber Bragg gratings [7653-96] H. K. Bal, Z. Brodzeli, F. Sidiroglou, S. F. Collins, Victoria Univ. (Australia)

$76530 Q \quad$ Rapid disappearance of regenerated fibre Bragg gratings at temperatures approaching $1500{ }^{\circ} \mathrm{C}$ in boron-codoped germanosilicate optical fibre [7653-106] M. L. Åslund, J. Canning, The Univ. of Sydney (Australia); H. Fu, H. Tam, The Hong Kong Polytechnic Univ. (Hong Kong, China)

7653 OR Two-dimensional fibre grating packaging design for simultaneous strain and temperature measurement [7653-131]

M. R. Mokhtar, City Univ. London (United Kingdom) and Multimedia Univ. (Malaysia); T. Sun, K. T. V. Grattan, City Univ. London (United Kingdom) 
7653 OS Frequency response of underwater ultrasonic transducers in the near field using polarimetric polarization maintaining fiber sensors [7653-157]

A. Bennecer, M. McGuire, G. M. H. Flockhart, S. G. Pierce, G. Hayward, B. Culshaw, Univ. of Strathclyde (United Kingdom)

7653 OT Dynamic studies of fibre Bragg gratings [7653-07]

D. A. Jackson, Univ. of Kent (United Kingdom)

$7653 \mathrm{OU} \quad$ Understanding the mode coupling process in a strong long period grating [7653-21]

L. Jin, W. Jin, J. Ju, The Hong Kong Polytechnic Univ. (Hong Kong, China)

7653 OV Fiber optic Bragg grating sensors at cryogenic temperatures [7653-55]

T. Habisreuther, W. Ecke, I. Latka, K. Schröder, R. Willsch, IPHT Jena (Germany)

7653 OW An ultra-high-resolution FBG static-strain sensor for geophysics applications [7653-97]

Q. Liu, Z. He, T. Tokunaga, K. Hotate, The Univ. of Tokyo (Japan)

7653 OX Temperature and strain independent torsion sensor using a Sagnac interferometer based on a suspended twin-core fibre [7653-11]

R. M. Silva, INESC Porto (Portugal); J. Kobelke, K. Schuster, IPHT Jena (Germany); O. Frazão, INESC Porto (Portugal)

7653 OY Acoustically modulated long period grating sensor for simultaneous viscosity and density measurement [7653-16]

R. A. Oliveira, Univ. Tecnológica Federal do Paraná (Brazil) and The Univ. of Sydney

(Australia); M. Naqshbandi, K. Cook, J. Canning, The Univ. of Sydney (Australia); A. A. P. Pohl, Univ. Tecnológica Federal do Paraná (Brazil)

$7653 \mathrm{OZ}$ Dual polarization fiber grating laser accelerometer [7653-19]

B.-O. Guan, Jinan Univ. (China) and Dalian Univ. of Technology (China); X.-S. Sun, Y.-N. Tan, Dalian Univ. of Technology (China)

765310 Multi-parameter sensor using fiber in-line MZ interferometer embedded in fiber Bragg grating [7653-25]

C. R. Liao, Y. Wang, D. N. Wang, M. Yang, The Hong Kong Polytechnic Univ. (Hong Kong, China)

765311 Intrinsic Fabry-Pérot cavity sensor based on chemical etching of a multimode graded index fiber spliced to a single mode fiber [7653-76]

P. A. R. Tafulo, O. Frazão, P. A. S. Jorge, INESC Porto (Portugal); F. M. Araújo, INESC Porto

(Portugal) and FiberSensing (Portugal)

765312 Strongly regenerated Bragg gratings in standard single-mode fibres [7653-22]

V. de Oliveira, H. J. Kalinowski, Univ. Tecnológica Federal do Paraná (Brazil)

765313 Optical vibration sensor using FBG Fabry-Perot interferometer with wavelength scanning and Fourier analysis [7653-67]

A. Wada, S. Tanaka, N. Takahashi, National Defense Academy (Japan) 
765314 A fibre Bragg grating-based inclinometer system for ground movement measurement [7653-74]

J. Li, R. Correia, E. Chehura, S. Staines, S. W. James, R. P. Tatam, Cranfield Univ. (United Kingdom)

765315 High sensitivity inline fiber Mach-Zehnder interferometer bend sensor using a twin core fiber [7653-138]

A. Harhira, J. Lapointe, R. Kashyap, Ecole Polytechnique de Montréal (Canada)

765316 Optical fibre sensor for measurement of the large range of strain and frequency vibrations of flat diaphragm [7653-152]

H. Krisch, M. Lau, S. Tournillon, Krohne Messtechnik GmbH \& Co. KG (Germany)

765317 Simulation of vibration induced changes in the fiber Bragg grating reflection spectrum [7653-141]

A. Aray, H. Saghafifar, Malek-e-Ashtar Univ. of Technology (Iran, Islamic Republic of)

765318 Angle transducer based on fiber Bragg gratings able for tunnel auscultation [7653-60] A. Quintela, J. M. Lázaro, M. A. Quintela, J. Mirapeix, V. Muñoz-Berti, J. M. López-Higuera, Univ. de Cantabria (Spain)

765319 Reflective polarimetric fiber optic thermometer [7653-102]

R. Wüest, ABB Ltd. (Switzerland); B. Gülenaltin, ABB Ltd. (Switzerland) and ETH Zurich (Switzerland); F. Buchter, T. Bühler, ABB Ltd. (Switzerland)

76531 A Dynamic response of optical fiber Bragg grating temperature sensors [7653-137] G. E. da Silva, J. C. Santos, Univ. of São Paulo (Brazill); V. R. de Almeida, R. M. Caso, Instituto de Estudos Avançados (Brazil)

Thursday Morning, 9 September 2010

SESSION II CHEMICAL, ENVIRONMENTAL, BIOMECHANICAL, AND MEDICAL SENSORS

7653 1B Microstructured optical fiber with homogeneous monolayer of plasmonic nanoparticles for bioanalysis [7653-36]

K. Schröder, A. Csaki, I. Latka, T. Henkel, D. Malsch, K. Schuster, T. Schneider, D. Zopf, IPHT Jena (Germany)

$76531 \mathrm{C}$ Diffuse-light absorption spectroscopy by fiber optics for detecting and quantifying the adulteration of extra virgin olive oil [7653-45]

A. G. Mignani, L. Ciaccheri, IFAC-CNR (Italy); H. Ottevaere, H. Thienpont, Vrije Univ. Brussel (Belgium); L. Conte, M. Marega, Univ. degli Studi di Udine (Italy); A. Cichelli, Univ. degli Studi G. D'Annunzio (Italy); C. Attilio, A. Cimato, IVALSA-CNR (Italy)

7653 ID Ammonia sensing using a fibre optic long period grating with a porous nanostructured coating formed from silica nanospheres [7653-73]

S. Korposh, Cranfield Univ. at Kitakyushu (Japan) and The Univ. of Kitakyushu (Japan); W. Batty, Cranfield Univ. at Kitakyushu (Japan); S. Kodaira, S.-W. Lee, The Univ. of Kitakyushu (Japan); S. W. James, S. M. Topliss, R. P. Tatam, Cranfield Univ. (United Kingdom) 
7653 1E Lab-on-a-fiber: building a fiber optic sensing platform for low-cost and high-performance trace vapor TNT detection [7653-15]

J. Ma, A. Kos, W. J. Bock, Univ. du Québec en Outaouais (Canada); X. Li, H. Nguyen,

Z. Y. Wang, Carleton Univ. (Canada); A. Cusano, Univ. degli Studi del Sannio (Italy)

$7653 \mathrm{IF} \quad$ Moving the wavelength detection range in surface plasmon resonance sensors based on tapered optical fibers [7653-44]

N. Díaz-Herrera, A. González-Cano, Univ. Complutense de Madrid (Spain); D. Viegas,

J. L. Santos, INESC Porto (Portugal) and Univ. do Porto (Portugal); M.-C. Navarrete, Univ.

Complutense de Madrid (Spain); Ó. Esteban, Univ. de Alcalá (Spain)

$76531 \mathrm{G} \quad$ Evanescent-wave LPFG in D-fiber by periodically patterned overlay [7653-75]

G. Quero, A. Crescitelli, D. Paladino, M. Consales, Univ. degli Studi del Sannio (Italy);

A. Buosciolo, M. Giordano, National Research Council (Italy); A. Cusano, Univ. degli Studi del Sannio (Italy)

$76531 \mathrm{H} \quad$ A portable instrument for the optical interrogation of a novel biochip [7653-128]

F. Baldini, IFAC-CNR (Italy); L. Bolzoni, Datamed S.r.L. (Italy); A. Giannetti, IFAC- CNR (Italy);

G. Porro, Datamed S.r.L. (Italy); C. Trono, IFAC-CNR (Italy)

$765311 \quad$ Optical fiber biosensor based on enzymatic coating matrix for catecholamines assessment in human urine [7653-31]

L. I. B. Silva, Instituto Piaget (Portugal) and Univ. de Aveiro (Portugal); A. C. Freitas,

T. A. P. Rocha-Santos, Instituto Piaget (Portugal); M. E. Pereira, A. C. Duarte, Univ. de Aveiro (Portugal)

$76531 \mathrm{~J} \quad$ Spectral marks for qualitative discriminant analysis [7653-33]

O. M. Conde, L. Uriarte, P. B. García-Allende, A. M. Cubillas, F. Anabitarte,

J. M. Lopez-Higuera, Univ. de Cantabria (Spain)

$76531 \mathrm{~K}$ Comparison of 2D planar approximation and rigorous 3D theoretical analysis of a fiber optic surface plasmon resonance sensor utilizing a Bragg grating [7653-149]

B. Špačková, J. Homola, Institute of Photonics and Electronics of the ASCR, v.v.i. (Czech Republic)

$76531 \mathrm{~L} \quad$ Regenerated Bragg gratings in high birefringence optical fibers [7653-71]

I. Abe, Univ. de Aveiro (Portugal); V. Oliveira, H. J. Kalinowski, Univ. Tecnológica Federal do Paraná (Brazil); J. L. Pinto, Univ. de Aveiro (Portugal)

7653 1M Fabrication of dual analyte luminescent optrodes by photopolymerization [7653-150] P. A. S. Jorge, INESC Porto (Portugal); C. Maule, INESC Porto (Portugal) and Univ. do Porto (Portugal); O. Soppera, Institut de Sciences des Matériaux de Mulhouse, CNRS (France); P. V. S. Marques, INESC Porto (Portugal) and Univ. do Porto (Portugal)

$76531 \mathrm{~N}$ Optical cavity fibre sensor for detection of microcystin-LR in water [7653-13]

R. B. Queirós, INESC Porto (Portugal) and Univ. do Porto (Portugal); S. O. Silva, INESC Porto (Portugal); M. G. F. Sales, REQUIMTE, Instituto Superior de Engenharia do Porto (Portugal); J. P. Noronha, REQUIMTE, FCT-UNL (Portugal); O. Frazão, P. A. S. Jorge, G. G. Aguilar, INESC Porto (Portugal) 
765310 Functional multilayer coated long period grating tuned in transition region for life science applications [7653-48]

P. Pilla, Univ. degli Studi del Sannio (Italy); V. Malachovská, A. Borriello, M. Giordano,

L. Ambrosio, National Research Council (Italy); A. Cutolo, A. Cusano, Univ. degli Studi del Sannio (Italy)

7653 IP Optical properties of hydrogel-filled hollow core photonic crystal fibres [7653-135]

M. Rutowska, P. Galvin, F. C. Garcia Gunning, Univ. College Cork (Ireland)

$76531 Q \quad$ Remote gaseous acid sensing within a porphyrin-doped $\mathrm{TiO}_{2}$ sol-gel layer inside a structured optical fibre [7653-156]

G. Huyang, J. Canning, M. L. Åslund, M. Naqshbandi, D. Stocks, M. J. Crossley, The Univ. of Sydney (Australia)

7653 IR Ambient index sensor using a Sagnac interferometer based on a D-shaped polarization maintaining fiber [7653-35]

O.-J. Kwon, Y. B. Shim, R. K. Kim, Y.-G. Han, Hanyang Univ. (Korea, Republic of)

7653 is Characterization of nanolayer LPG refractometer according surrounding refractive index [7653-63]

E. Simões, I. Abe, J. Oliveira, J. L. Pinto, Univ. de Aveiro (Portugal); P. Caldas, INESC Porto (Portugal) and Escola Superior de Tecnologia e Gestão (Portugal); O. Frazão, INESC Porto (Portugal)

7653 IT POF metrology in physics medicine and rehabilitation [7653-65]

L. Bilro, Univ. de Aveiro (Portugal); J. G. Oliveira, Hospital Inf. D. Pedro (Portugal); J. L. Pinto, Univ. de Aveiro (Portugal); R. N. Nogueira, Instituto de Telecomunicações (Portugal)

7653 iU A cylindrical-core fibre optic oxygen sensor based on Pt (II) complexes immobilized in a polymer matrix [7653-1 11 l]

R. Chen, A. D. Farmery, Univ. of Oxford (United Kingdom); A. Obeid, Oxford Optronix Ltd. (United Kingdom); C. E. W. Hahn, Univ. of Oxford (United Kingdom)

7653 IV A fibre optic chemical sensor for the detection of cocaine [7653-146] T. H. Nguyen, T. Sun, K. T. V. Grattan, City Univ. London (United Kingdom); S. A. Hardwick, Home Office Scientific Development Branch (United Kingdom)

7653 IW Lossy-mode resonance-based refractometers by means of indium oxide coatings fabricated onto optical fibers [7653-61]

C. R. Zamarreño, I. Del Villar, P. Sanchez, M. Hernaez, C. Fernandez, I. R. Matias, F. J. Arregui, Univ. Pública de Navarra (Spain)

7653 1X High sensitivity transition-tuned long period grating for label-free immunosensing [7653-59] P. Pilla, Univ. degli Studi del Sannio (Italy); V. Malachovská, A. Sandomenico, M. Ruvo, M. Giordano, National Research Council (Italy); A. Cutolo, A. Cusano, Univ. degli Studi del Sannio (Italy) 
7653 IY Self-assembling and coordination of water nano-layers on polymeric coated long period gratings as promising tool for cation detection [7653-119]

P. Foglia Manzillo, Univ. degli Studi di Napoli Parthenope (Italy); P. Pilla, Univ. degli Studi del Sannio (Italy); S. Campopiano, Univ. degli Studi di Napoli Parthenope (Italy); A. Borriello, M. Giordano, National Research Council (Italy); A. Cusano, Univ. degli Studi del Sannio (Italy)

$765312 \quad$ Femtosecond laser induced microfiber Bragg grating for refractive index sensing [7653-26] C. R. Liao, X. Fang, D. N. Wang, The Hong Kong Polytechnic Univ. (Hong Kong, China)

765320 Humidity sensor based on a long-period fiber grating coated with a hydrophobic thin film [7653-29]

A. Urrutia, P. J. Rivero, J. Goicoechea, F. J. Arregui, I. R. Matías, Univ. Pública de Navarra (Spain)

765321 Colloidal-core photonic crystal fiber incorporating CdSe quantum dots for temperature sensing [7653-116]

A. Bozolan, Univ. de São Paulo (Brazil) and Univ. Presbiteriana Mackenzie (Brazil);

C. J. S. de Matos, Univ. Presbiteriana Mackenzie (Brazil); M. A. Romero, Univ. de São Paulo (Brazil)

765322 Biconical tapered optical fiber biosensor for real-time monitoring of bovine serum albumin at femtogram $/ \mathrm{mL}$ levels on antibody-immobilized tapered fibers [7653-41]

M. I. Zibaii, H. Latifi, M. Arabsorkhi, A. Kazemi, M. Gholami, M. Karimi Azar, S. M. Hosseini,

Shahid Beheshti Univ. (Iran, Islamic Republic of)

765323 Fiber optic biosensor fabricated for measuring the growth rate of Escherichia coli K-12 in the aqueous [7653-42]

M. I. Zibaii, A. Kazemi, H. Latifi, M. Karimi Azar, S. M. Hosseini, M. H. Ghezelaiagh, Shahid

Beheshti Univ. (Iran, Islamic Republic of)

765324 Simultaneous strain and refractive index sensor based on a TFBG [7653-79]

N. J. Alberto, Univ. de Aveiro (Portugal); C. A. Marques, Instituto de Telecomunicações

(Portugal); J. L. Pinto, Univ. de Aveiro (Portugal); R. N. Nogueira, Instituto de

Telecomunicações (Portugal)

765325 Optical fiber micro-analyzer for real-time monitoring of trimethylamine [7653-32]

L. I. B. Silva, Instituto Piaget (Portugal) and Univ. de Aveiro (Portugal); A. C. Freitas,

T. A. P. Rocha-Santos, Instituto Piaget (Portugal); M. E. Pereira, A. C. Duarte, Univ. de Aveiro (Portugal)

765326 Operation of optical fiber sensors in hydrogen-rich atmosphere [7653-144]

C. Martelli, Univ. Tecnológica Federal do Paraná (Brazil); A. L. C. Triques, Petrobras Research Ctr. (Brazil); A. Braga, Pontifícia Univ. Católica do Rio de Janeiro (Brazil); J. Canning, K. Cook, The Univ. of Sydney (Australia); R. Llerena, V. Takahashi, Pontifícia Univ. Católica do Rio de Janeiro (Brazil) 
765327 Low cost plastic optical fiber sensor based on surface plasmon resonance [7653-148] V. M. Muñoz-Berti, A. C. López-Pérez, Instituto de Microelectrónica de Madrid (Spain) and Univ. de Cantabria (Spain); B. Alén, J. L. Costa-Krämer, A. García-Martín, Instituto de Microelectrónica de Madrid (Spain); M. Lomer, J. M. López-Higuera, Univ. de Cantabria (Spain)

765328 Low power signal processing for demodulation of wide dynamic range of interferometric optical fibre sensor signals [7653-153]

N. Fernandes, Krohne Marshall Pvt Ltd. (India); K. Gossner, H. Krisch, Krohne Messtechnik GmbH \& Co. KG (Germany)

765329 Sensing applications of U-optrodes [7653-57]

L. Kalvoda, J. Aubrecht, R. Klepáček, P. Lukášová, Czech Technical Univ. in Prague (Czech Republic)

Thursday Afternoon, 9 September 2010

SESSION III ELECTROMAGNETIC, INTERFEROMETRIC, POLARIMETRIC, NEW CONCEPTS, AND DEVICES FOR SENSORS

7653 2A Temperature- and strain-independent curvature sensor based on multimode interference [7653-14]

S. Silva, INESC Porto (Portugal) and Univ. do Porto (Portugal); O. Frazão, L. A. Ferreira,

F. M. Araújo, INESC Porto (Portugal); F. X. Malcata, Instituto Superior da Maia (Portugal) and Ctr. Interdisciplinar de Investigação Marinha e Ambiental (Portugal); J. L. Santos, INESC Porto (Portugal) and Univ. do Porto (Portugal)

$76532 \mathrm{~B} \quad$ Enhancing absorption and sensitivity within structured optical fibres [7653-52]

J. Canning, W. Padden, D. Boskovic, M. Naqshbandi, L. Costanzo, G. Huyang, H. de Bruyn,

T. H. Sum, M. J. Crossley, The Univ. of Sydney (Australia)

7653 2C Dynamic interrogation of long period gratings with modulated fibre Bragg gratings [7653-121]

J. P. Carvalho, INESC Porto (Portugal) and Univ. do Porto (Portugal); L. Coelho, INESC Porto (Portugal); L. Correia, Instituto Militar de Engenharia (Brazil); O. Frazão, P. A. S. Jorge, INESC Porto (Portugal); J. M. Baptista, INESC Porto (Portugal) and Univ. da Madeira (Portugal);

I. Dias, INESC Porto (Portugal); J. L. Santos, INESC Porto (Portugal) and Univ. do Porto (Portugal); J. Weyl, Univ. Federal do Pará (Brazil); M. J. Pontes, Univ. Federal do Espírito Santo (Brazil); M. A. Martinez, Ctr. Federal de Educação Tecnológica Celso Suckow de Fonesca (Brazil); A. P. Barbero, R. M. Ribeiro, Univ. Federal Fluminense (Brazil); A. J. G. Abelém, Univ. Federal do Pará (Brazil); M. T. R. Giraldi, Instituto Militar de Engenharia (Brazil)

$76532 \mathrm{D}$ Feedback controlled single wavelength interrogation technique for miniature all-silica EFPI fibre optic pressure sensors [7653-125]

K. Bremer, E. Lewis, G. Leen, B. Moss, Univ. of Limerick (Ireland); S. Lochmann, I. Mueller, Hochschule Wismar (Germany)

$76532 \mathrm{E}$ Digital control of a white light system for interrogation of optical fiber interferometric sensors [7653-129]

E. Velosa, C. Gouveia, Univ. da Madeira (Portugal) and INESC Porto (Portugal);

P. A. S. Jorge, INESC Porto (Portugal); J. M. Baptista, Univ. da Madeira (Portugal) 
$76532 \mathrm{~F}$ Fibre optic remote sensing based on long period gratings with in situ optical source [7653-122]

D. Viegas, J. P. Carvalho, Univ. do Porto (Portugal) and INESC Porto (Portugal); L. Coelho, INESC Porto (Portugal); J. L. Santos, Univ. do Porto (Portugal) and INESC Porto (Portugal); L. A. Ferreira, F. M. Araújo, O. Frazão, INESC Porto (Portugal)

$76532 \mathrm{G}$ All-fibre interferometric configurations based on suspended-core fibres for pressure measurement [7653-30]

S. H. Aref, INESC Porto (Portugal), Univ. of Qom (Iran, Islamic Republic of), and Shahid Beheshti Univ. (Iran, Islamic Republic of); M. I. Zibaii, M. Kheiri, H. Porbeyram, H. Latifi, Shahid Beheshti Univ. (Iran, Islamic Republic of); O. Frazão, F. M. Araújo, L. A. Ferreira, INESC Porto (Portugal); J. L. Santos, INESC Porto (Portugal) and Univ. do Porto (Portugal); J. Kobelke, K. Schuster, IPHT Jena (Germany)

$76532 \mathrm{H} \quad$ Bragg gratings in the germanium-doped concentric rings of an $\mathrm{Yb}^{3+}$-doped core solid photonic bandgap fibre [7653-69]

K. Cook, W. Padden, J. Canning, The Univ. of Sydney (Australia); S. Février, XLIM, CNRS, Univ. de Limoges (France); B. Li, The Univ. of Sydney (Australia) and Yanshan Univ. (China)

$765321 \quad$ Magnetic field strength measurement using tailored dispersion characteristics of phaseshifted fibre Bragg gratings [7653-24]

P. Orr, Univ. of Strathclyde (United Kingdom); M. Stevenson, J. Canning, The Univ. of Sydney (Australia); P. Niewczas, Univ. of Strathclyde (United Kingdom)

7653 2J Surface-plasmon-resonance sensor based on H-shaped optical fibre [7653-104] D. Viegas, Univ. do Porto (Portugal) and INESC Porto (Portugal); M. Hautakorpi, Aalto Univ. (Finland); A. Guerreiro, J. L. Santos, Univ. do Porto (Portugal) and INESC Porto (Portugal); H. Ludvigsen, Aalto Univ. (Finland)

$76532 \mathrm{~K}$ Fiber optic intensity sensor referenced with virtual instrumentation for measuring displacement [7653-90]

A. J. G. Fernandes, Univ. da Madeira (Portugal); C. Jesus, Univ. da Madeira (Portugal) and INESC Porto (Portugal); P. A. S. Jorge, INESC Porto (Portugal); J. M. Baptista, Univ. da Madeira (Portugal) and INESC Porto (Portugal)

$76532 \mathrm{M} \quad$ A Raman intensity sensor induced by the Rayleigh scattering in a ring configuration [7653-50]

C. Correia, J. M. Baptista, INESC Porto (Portugal) and Univ. da Madeira (Portugal); M. B. Marques, INESC Porto (Portugal) and Univ. do Porto (Portugal); O. Frazão, INESC Porto (Portugal)

$76532 \mathrm{~N}$ Acoustic source location of partial discharges in transformers [7653-143] S. E. U. Lima, O. Frazão, INESC Porto (Portugal); R. G. Farias, Univ. Federal do Pará (Brazil); F. M. Araújo, L. A. Ferreira, INESC Porto (Portugal); V. Miranda, J. L. Santos, INESC Porto (Portugal) and Univ. do Porto (Portugal)

765320 An all-fiber beam shaping using multimode fiber [7653-92] M. Mayeh, F. Farahi, The Univ. of North Carolina at Charlotte (United States) 
$76532 \mathrm{P} \quad$ Interrogation of microresonators using multimode fibers [7653-81]

P. Caldas, INESC Porto (Portugal), Univ. do Porto (Portugal), and Escola Superior de Tecnologia e Gestão (Portugal); P. A. S. Jorge, F. A. Araújo, L. A. Ferreira, INESC Porto (Portugal); G. Rego, INESC Porto (Portugal) and Escola Superior de Tecnologia e Gestão (Portugal); J. L. Santos, INESC Porto (Portugal) and Univ. do Porto (Portugal); S. Berneschi, Ctr. Studi e Ricerche Enrico Fermi (Italy) and IFAC-CNR (Italy); F. Cosi, S. Soria, S. Pelli, G. Nunzi Conti, IFAC-CNR (Italy)

$76532 \mathrm{Q}$ Fiber optic simultaneous measurement of strain and temperature using a PM-PCF-based Sagnac interferometer incorporating a pumped EDF [7653-08] O.-J. Kwon, H.-J. Kim, S. Cuh, M.-S. Yoon, S. Park, Y. Shim, Hanyang Univ. (Korea, Republic of); M. Y. Jeong, C.-S. Kim, Pusan National Univ. (Korea, Republic of); Y.-G. Han, Hanyang Univ. (Korea, Republic of)

$76532 \mathrm{R}$ Residual thermal stress-induced integrated optical waveguides on $\mathrm{Bi}_{12} \mathrm{GeO}_{20}$ substrate [7653-136]

S. S. Sato, J. C. Santos, Univ. de São Paulo (Brazil); M. A. R. Franco, Instituto de Estudos Avançados (Brazil)

765325 Analysis and realization of a fiber optic turbidity sensor based on measurements of scattered light [7653-124]

Prerana, M. R. Shenoy, B. P. Pal, B. D. Gupta, Indian Institute of Technology Delhi (India)

Friday, 10 September 2010

SESSION IV DISTRIBUTED, MULTIPLEXING, SYSTEM APPLICATIONS, FIELD TRIALS

$76532 \mathrm{~T} \quad$ Measurement with $2 \mathrm{~m}$ resolution using a Raman-assisted BOTDA sensor featuring $75 \mathrm{~km}$ dynamic range [7653-120]

S. Martin-Lopez, F. Rodriguez-Barrios, Consejo Superior de Investigaciones Científicas (Spain); A. Carrasco-Sanz, Univ. de Granada (Spain); P. Corredera, J. D. Ania-Castañon, Consejo Superior de Investigaciones Científicas (Spain); L. Thevenaz, Ecole Polytechnique Fédérale de Lausanne (Switzerland); M. Gonzalez-Herraez, Univ. de Alcalá (Spain)

$76532 \mathrm{U} \quad$ Impact of self phase modulation on the performance of Brillouin distributed fibre sensors [7653-140]

S. Foaleng Mafang, Ecole Polytechnique Fédérale de Lausanne (Switzerland); F. Rodríguez, S. Martin Lopez, Consejo Superior de Investigaciones Científicas (Spain);

M. González Herráez, Univ. de Alcalá (Spain); L. Thévenaz, Ecole Polytechnique Fédérale de Lausanne (Switzerland)

$76532 \mathrm{~V} \quad$ A novel fiber optic technique for quasi-distributed and dynamic measurement of length change and refractive index [7653-72]

S. Liehr, K. Krebber, Bundesanstalt für Materialforschung und -prüfung (Germany)

7653 2W Distributed gas sensor based on a photonic bandgap fiber cell with laser-drilled, lateral microchannels [7653-118]

H. Lehmann, H. Bartelt, R. Willsch, IPHT Jena (Germany); R. Amezcua-Correa, J. C. Knight, Univ. of Bath (United Kingdom) 
$76532 Y \quad$ Distributed optical fibre sensing of temperature using time-correlated two-photon excited fluorescence [7653-88]

C. J. Dalzell, T. P. J. Han, I. S. Ruddock, Univ. of Strathclyde (United Kingdom)

$76532 Z$ A new MRI-compatible optical fiber tactile sensor for use in minimally invasive robotic surgery systems [7653-49]

R. Ahmadi, J. Dargahi, M. Packirisamy, Concordia Univ. (Canada); R. Cecere, McGill Univ. (Canada)

765330 Measurement of the longitudinal and circumferential muscular activity associated with peristalsis using a single fibre grating array [7653-68]

J. W. Arkwright, N. G. Blenman, I. D. Underhill, S. A. Maunder, Commonwealth Scientific and Industrial Research Organisation (Australia); N. J. Spencer, M. Costa, S. J. Brooks, Flinders Univ. (Australia); M. M. Szczesniak, P. G. Dinning, The Univ. of New South Wales (Australia)

765331 A fiber optics textile composite sensor for geotechnical applications [7653-23] O. Artières, TenCate Geosynthetics (France); G. Dortland, TenCate Geosynthetics (Netherlands)

765332 Impact of the pulse modulation format on distributed BOTDA sensors based on Simplex coding [7653-77]

M. A. Soto, G. Bolognini, F. Di Pasquale, Scuola Superiore Sant'Anna (Italy)

765333 Strain measurements of a fiber loop rosette using high spatial resolution Rayleigh scatter distributed sensing [7653-147]

D. K. Gifford, A. K. Sang, S. T. Kreger, M. E. Froggatt, Luna Technologies (United States)

765334 Low-cost Brillouin optical time domain analysis (BOTDA) distributed sensor setup [7653-80] A. Zornoza, A. Loayssa, Univ. Pública de Navarra (Spain)

765335 Fibre laser sensor based on a phase-shifted chirped grating for acoustic sensing of partial discharges in power transformers [7653-28]

S. E. U. Lima, INESC Porto (Portugal) and Univ. do Porto (Portugal); O. Frazão, INESC Porto (Portugal); R. G. Farias, Univ. Federal do Pará (Brazil); F. M. Araújo, L. A. Ferreira, INESC Porto (Portugal); V. Miranda, J. L. Santos, INESC Porto (Portugal) and Univ. do Porto (Portugal)

765336 Experimental study for the detection of the laminar/turbulent aerodynamic transition on a wing aircraft, using fiber optic sensors [7653-39]

S. Molin, D. Dolfi, Thales Research \& Technology (France); M. Doisy, Thales Underwater Systems (France); A. Seraudie, D. Arnal, E. Coustols, ONERA (France); J. Mandle, Thales Avionics (France)

765337 Fiber optic sensor solutions for increase of efficiency and availability of electric power generators [7653-113]

M. Willsch, T. Bosselmann, M. Villnow, Siemens AG (Germany)

765338 Ultrasonic modal detection in carbon fibre plates using fibre optic sensors [7653-117]

G. Thursby, B. Culshaw, Univ. of Strathclyde (United Kingdom) 
765339 Suspended-core Fabry-Perot temperature sensor interrogation through a dual wavelength Raman fiber laser [7653-04]

A. M. R. Pinto, Univ. Pública de Navarra (Spain); O. Frazão, INESC Porto (Portugal);

J. L. Santos, INESC Porto (Portugal) and Univ. do Porto (Portugal); M. Lopez-Amo, Univ.

Pública de Navarra (Spain); J. Kobelke, K. Schuster, IPHT Jena (Germany)

7653 3A Polymer photonic sensing skin [7653-89]

X. Chen, C. Zhang, D. J. Webb, Aston Univ. (United Kingdom); B. Van Hoe,

G. Van Steenberge, Ghent Univ. (Belgium); K. Kalli, Cyprus Univ. of Technology (Cyprus); F. Berghmans, H. Thienpont, Vrije Univ. Brussel (Belgium); W. Urbanczyk, Wroclaw Univ. of Technology (Poland); K. Sugden, Astasense Ltd. (United Kingdom); G.-D. Peng, The Univ. of New South Wales (Australia)

7653 3B Smart medical textiles with embedded optical fibre sensors for continuous monitoring of respiratory movements during MRI [7653-115]

J. Witt, Bundesanstalt für Materialforschung und -prüfung (Germany); F. Narbonneau, Multitel (Belgium); M. Schukar, K. Krebber, Bundesanstalt für Materialforschung und -prüfung (Germany); J. De Jonckheere, M. Jeanne, Ctr. Hospitalier Regional Univ. de Lille (France); D. Kinet, Multitel (Belgium); B. Paquet, Centexbel (Belgium); A. Depré, Elasta (Belgium); L. T. D'Angelo, Technische Univ. München (Germany); T. Thiel, AOS GmbH (Germany); R. Logier, Ctr. Hospitalier Regional Univ. de Lille (France)

7653 3C Smart CFRP systems for the controlled retrofitting of reinforced concrete members [7653-12] M.-B. Schaller, Gesellschaft für Geomechanik und Baumeßtechnik mbH (Germany); S. Käseberg, Hochschule für Technik, Wirtschaft und Kultur Leipzig (Germany); M. Kuhne, Bauhaus Univ. Weimar (Germany)

7653 3D Extension of the maximum measuring range in distributed Brillouin fiber sensors by tuning the Stokes/anti-Stokes power ratio [7653-56]

A. Minardo, Second Univ. of Naples (Italy); R. Bernini, Istituto per il Rilevamento Elettromagnetico dell'Ambiente, CNR (Italy); L. Zeni, Second Univ. of Naples (Italy) and Consortium for Research on Advanced Remote Sensing Systems (Italy)

7653 3E Distributed crystal fibre sensing for extreme environments [7653-87]

C. J. Dalzell, T. P. J. Han, I. S. Ruddock, Univ. of Strathclyde (United Kingdom)

$76533 \mathrm{~F} \quad$ Self-referenced wavelength-sensitive monitoring technique for quasi-distributed fiber Bragg gratings sensors [7653-95]

C. Caucheteur, D. Kinet, N. Ben Soltana, P. Mégret, M. Wuilpart, Univ. de Mons (Belgium)

7653 3G Fiber optic sensors for CMS-CERN [7653-05]

A. Cusano, Univ. degli Studi del Sannio (Italy) and OptoSmart s.r.I. (Italy); G. Breglio, OptoSmart s.r.l. (Italy) and Univ. degli Studi di Napoli Federico II (Italy); A. Irace, Univ. degli Studi di Napoli Federico II (Italy); M. Consales, Univ. degli Studi del Sannio (Italy); A. Buosciolo, Institute for Composite and Biomedical Materials, CNR (Italy); M. Giordano, Institute for Composite and Biomedical Materials, CNR (Italy) and Optosmart s.r.l. (Italy); A. Cutolo, Univ. degli Studi del Sannio (Italy) and OptoSmart s.r.l. (Italy); S. Buontempo, Istituto Nazionale di Fisica Nucleare (Italy); P. Petagna, European Organization for Nuclear Research (Switzerland) 
$76533 \mathrm{H} \quad$ Automated suppression of polarization-fluctuation in resonator fiber optic gyro by a resonator with twin $90^{\circ}$ polarization-axis rotated splices: theoretical analysis [7653-70] X. Wang, Z. He, K. Hotate, The Univ. of Tokyo (Japan)

$765331 \quad$ Fiber optic sensor network for monitoring new building cladding systems [7653-100] R. Unzu, J. A. Nazabal, Univ. Pública de Navarra (Spain); G. Vargas, Alonso, Hernández \& Associates Architects (Spain); R. Hernández, Univ. del País Vasco (Spain);

C. Fernández-Valdivielso, N. Urriza, M. Galarza, M. Lopez-Amo, Univ. Pública de Navarra (Spain)

7653 3J Unsupervised grouping of industrial textile dyes using K-means algorithm and optical fibre spectroscopy [7653-109]

A. M. Cubillas, O. M. Conde, P. Anuarbe, A. Quintela, J. M. Lopez-Higuera, Univ. de Cantabria (Spain)

$76533 \mathrm{~K}$ A process for embedding fiber Bragg gratings in flexible skin foils [7653-18]

A. F. Silva, Univ. of Minho (Portugal); F. Goncalves, TMG Automotive (Portugal); L. A. Ferreira, F. M. Araujo, FiberSensing (Portugal) and INESC Porto (Portugal); P. M. Mendes, J. H. Correia, Univ. of Minho (Portugal)

$76533 \mathrm{~L} \quad$ Low-cost self-referenced all-fibre polarimetric current sensor for the monitoring of current in the railway catenary [7653-114]

M. L. Filograno, Univ. de Alcalá (Spain); P. Corredera, Consejo Superior de Investigaciones Científicas (Spain); L. Monteagudo Lerma, Ó. Esteban, M. González-Herráez, Univ. de Alcalá (Spain)

$76533 \mathrm{M}$ Real-time monitoring of railway traffic using fiber Bragg gratings [7653-130]

M. L. Filograno, A. Rodriguez-Barrios, Univ. de Alcalá (Spain); P. Corredera, S. Martin-Lopez, Consejo Superior de Investigaciones Científicas (Spain); M. Rodriguez-Plaza,

A. Andres-Alguacil, Administrador de Infraestructuras Ferroviarias (Spain);

M. Gonzalez-Herraez, Univ. de Alcalá (Spain)

$76533 \mathrm{~N} \quad$ How reliable do fibre Bragg grating patches perform as strain sensors? [7653-10]

V. G. Schlüter, N. Kusche, W. R. Habel, Bundesanstalt für Materialforschung und -prüfung (Germany)

765330 Fiber Bragg gratings for optical sensing (FIBOS) for an aerospace application [7653-17] R. L. Heredero, M. Frovel, H. Laguna, A. Anderson, D. Garranzo, T. Belenguer-Dávila, Instituto Nacional de Técnica Aeroespacial (Spain)

7653 3P Raman distributed temperature sensing in underground geoexchange system [7653-37] M. Giuseffi, P. Ferdinand, CEA, LIST, Lab. de Mesures Optiques (France); A. Vrain, M. Philippe, H. Lesueur, BRGM (France)

$76533 \mathrm{Q}$ An approach to monitor railway pantograph-catenary interaction with fiber optic sensors [7653-82]

M. Bocciolone, G. Bucca, A. Collina, L. Comolli, Politecnico di Milano (Italy) 
$76533 R \quad$ Long-range hybrid double-bus network with point and distributed Brillouin sensors using Raman amplification [7653-126]

M. Fernandez-Vallejo, D. Olier, A. Zornoza, R. A. Perez-Herrera, S. Díaz, C. Elosúa, C. Bariain, A. Loayssa, M. Lopez-Amo, Univ. Pública de Navarra (Spain)

7653 3S Permanently bent single mode optical fiber as novel evanescent wave sensor [7653-127] A. Iadicicco, Univ. degli Studi di Napoli Parthenope (Italy); D. Paladino, Univ. degli Studi del Sannio (Italy); S. Campopiano, Univ. degli Studi di Napoli Parthenope (Italy); W. Bock, Univ. du Québec en Outaouais (Canada); A. Cutolo, A. Cusano, Univ. degli Studi del Sannio (Italy)

7653 3T Design of a surface attachable hybrid fiber sensor packaged in a polyimide film for engineering applications [7653-142]

M. Ramakrishnan, G. Rajan, Y. Semenova, G. Farrell, Dublin Institute of Technology (Ireland)

$76533 \mathrm{U} \quad$ Location of vibrational disturbance using a serial array of identical low-finesse fiber FabryPerot interferometers [7653-145]

R. Martínez Manuel, Univ. of Johannesburg (South Africa); M. G. Shlyagin, S. V. Miridonov, Ctr. de Investigación Científica y de Educación Superior de Ensenada (Mexico); J. Meyer, Univ. of Johannesburg (South Africa)

7653 3V Stable multiwavelength fiber laser for referencing intensity sensor networks using multiple amplified ring resonators [7653-02]

M. Fernandez-Vallejo, R. A. Perez-Herrera, C. Elosúa, C. Bariain, M. Lopez-Amo, Univ. Pública de Navarra (Spain)

7653 3W Broadband photonic crystal fiber coupler with polarization selection of coupling ratio [7653-47]

L. R. Jaroszewicz, K. A. Stasiewicz, P. Marć, M. Szymański, Military Univ. of Technology (Poland)

7653 3X Distributed humidity sensing based on Rayleigh scattering in polymer optical fibers [7653-64] P. Lenke, M. Wendt, S. Liehr, K. Krebber, Bundesanstalt für Materialforschung und -prüfung (Germany)

7653 3Y Performance evaluation of an IGBT module by thermal analysis using fiber Bragg grating [7653-86]

J. P. Bazzo, T. Lukasievicz, M. Vogt, M. L. S. Martins, H. J. Kalinowski, J. C. C. Silva, Univ. Tecnológica Federal do Paraná (Brazil)

$765332 \quad$ Numerical modeling of a birefringent photonic crystal fiber for discrete and distributed pressure sensing [7653-91]

R. C. R. Miraglia, C. J. S. de Matos, Univ. Presbiteriana Mackenzie (Brazil)

765340 Hybrid OTDR-fiber laser system for remote sensor multiplexing [7653-101]

M. Bravo, M. Fernandez-Vallejo, M. Lopez-Amo, Univ. Publica de Navarra (Spain)

765341 An application of FBG accelerometers for monitoring pantographs of underground trains [7653-84]

M. Bocciolone, G. Bucca, A. Cigada, A. Collina, L. Comolli, Politecnico di Milano (Italy) 
765342 Single-mode distributed temperature sensing using OFDR [7653-53]

W. Hill, J. Kübler, M. Fromme, LIOS Technology GmbH (Germany)

765343 Multipoint dynamically reconfigure adaptive distributed fiber optic acoustic emission sensor (FAESense) system for condition based maintenance [7653-165]

E. Mendoza, J. Prohaska, C. Kempen, Y. Esterkin, S. Sun, Redondo Optics, Inc. (United States);

S. Krishnaswamy, Northwestern Univ. (United States)

765344 Bridge monitoring by Brillouin-based distributed strain measurements [7653-54]

A. Minardo, Second Univ. of Naples (Italy); R. Bernini, Istituto per il Rilevamento

Elettromagnetico dell'Ambiente, CNR (Italy); L. Amato, Tecno In S.p.A. (Italy); L. Zeni, Second Univ. of Naples (Italy) and Consortium for Research on Advanced Remote Sensing Systems (Italy)

765345 Use of fiber optic sensors for measurement of railway vibrations [7653-38]

A. Barreda, T. Molina, E. Valero, S. Recuero, AIDO-Instituto Tecnológico de Óptica, Color e Imagen (Spain)

765346 Comparison of optical and electrical measurements of the pantograph-catenary contact force [7653-83]

M. Bocciolone, G. Bucca, A. Collina, L. Comolli, Politecnico di Milano (Italy)

765347 Automatic classification of steel plates based on laser induced breakdown spectroscopy and support vector machines [7653-108]

F. Anabitarte, J. Mirapeix, O. M. Conde, A. M. Cubillas, L. Rodriguez-Cobo, C. Galindez, A. Cobo, Univ. de Cantabria (Spain)

Author Index 


\title{
Conference Committee
}

\author{
General Chair \\ José Luís Santos, University of Porto (Portugal) \\ Program Chairs
}

Brian Culshaw, University of Strathclyde (United Kingdom) José Miguel López-Higuera, University of Cantabria (Spain)

William N. MacPherson, Heriot-Watt University (United Kingdom)

Program Committee

Adolfo Cobo García, University of Cantabria (Spain)

Anders Bjarklev, Technical University of Denmark (Denmark)

António Lobo Ribeiro, University Fernando Pessoa (Portugal)

Andrea Cusano, University of Sannio (Italy)

Avishay Eyal, Tel-Aviv University (Israel)

Daniele Inaudi, Smartec (Switzerland)

Dominique Pagnoux, University of Limoges (France)

Elfed Lewis, University of Limerick (Ireland)

Francesco Baldini, IFAC-CNR (Italy)

George Stewart, University of Strathclyde (United Kingdom)

Guillermo Orellana, Complutense University of Madrid (Spain)

Hugo Thienpont, Vrije Universiteit Brussel (Belgium)

Katerina Krebber, Bundesanstalt für Materialforschung und -prüfung

(Germany)

Jan Rayss, Marie Curie-Skłodowska University (Poland)

Julian Jones, Heriot-Watt University (United Kingdom)

Leszek Jaroszewicz, Military University of Technology (Poland)

Ladislav Kalvoda, Czech Technical University in Prague

(Czech Republic)

Luís Alberto Ferreira, INESC Porto (Portugal)

Luc Thévenaz, Ecole Polytechnique Fédérale de Lausanne

(Switzerland)

Manuel Lopez-Amo, Public University of Navarra (Spain)

Mark Voet, FOS\&S (Belgium)

Marco Petrovich, University of Southampton (United Kingdom)

Mario Martinelli, University of Milan (Italy)

Reinhardt Willsch, IPHT-Jena (Germany)

Pierre Ferdinand, Commissariat à l'Énergie Atomique (France)

Stephen W. James, Cranfield University (United Kingdom)

Thomas Bosselmann, Siemens AG (Germany) 
Tong Sun, City University London (United Kingdom)

Waclaw Urbanczyk, Wroclaw University of Technology (Poland)

Walter Margulis, ACREO (Sweden)

Karsten Rottwitt, OSA Representative, Technical University of Denmark

(Denmark)

International Advisory Committee

Alexis Mendez, MCH Engineering (United States)

Anna Grazia Mignani, IFAC-CNR (Italy)

Robert Lieberman, Intelligent Optical Systems Inc. (United States)

Bishnu Pal, Indian Institute of Technology Delhi (India)

Byoung Yoon Kim, Korea Advanced Institute of Science and Technology (Korea, Republic of)

David Sampson, University of Western Australia (Australia)

Fernando Mendoza, Center for Research in Optics (Mexico)

Hypolito José Kalinowski, Federal University of Technology (Brazil)

Ian Bennion, Aston University (United Kingdom)

Kazuo Hotate, The University of Tokyo (Japan)

Jiř́ Homola, Institute of Photonics and Electronics (Czech Republic)

Moshe Tur, Tel-Aviv University (Israel)

Raman Kashyap, École Polytechnique de Montréal (Canada)

Yanbiao Liao, Tsinghua University (China)

Karsten Rottwitt, OSA Representative, Technical University of Denmark

(Denmark)

Local Organizing Committee

Ariel Guerreiro, University of Porto (Portugal)

Francisco M. Araújo, INESC Porto (Portugal)

Ireneu Dias, INESC Porto (Portugal)

José Manuel Baptista, University of Madeira (Portugal)

Orlando Frazão, INESC Porto (Portugal)

Paulo Marques, University of Porto (Portugal)

Pedro A. S. Jorge, INESC Porto (Portugal)

Sandra Pinto, INESC Porto (Portugal)

Administrative Local Organizing Committee

Sofia Santos, INESC Porto (Portugal)

Luísa Mendonça, INESC Porto (Portugal) 


\section{Introduction}

Welcome to Porto and to the Fourth European Workshop on Optical Fibre Sensors. Following the successful meetings in Peebles (1998), Santander (2004), and Napoli (2007), we have the privilege of organizing the 2010 meeting, EWOFS'2010.

The Workshop series aims to promote a scientific meeting with a high level of interaction between participants, enabling an open debate and the assessment of new concepts, technologies, and applications in the domain of optical fibre sensors, as well as the establishment of new collaborations and networks.

Addressing scientific achievements, technological applications, and commercial exploitation, our goal was to create a program that could be attractive to both academics and professionals working in this area.

We have tried to incorporate a number of high-level invited talks addressing not only topics related to optical fibre sensors, but also other scientific domains that may be relevant to the future of this R\&D field. Also, we wanted to strengthen the workshop's unique features, including the discussion of technical contributions, identifying and highlighting the most significant achievements. Because everyone's active participation is important, we planned a workshop with an invigorating and appealing social program that encourages true scientific socialization.

EWOFS' 2010 takes place a decade after the beginning of this new century, of this new millennium. Humankind is currently facing great challenges in the search for a future characterized by global justice, fair and sustainable progress, as well as economic and social wealth. This is a demanding, yet necessary purpose, and science and technology create opportunities so that society may evolve in that direction. A special session was organized on "Optical Fibre Sensors for Sustainable Environment" which reflects our commitment to one of the big challenges humankind currently faces.

EWOFS'2010 aims to encourage scientific and technological advances, and provide a forum where young and experienced researchers and entrepreneurs may interact in a mutually profitable relationship oriented towards the development of optical fibre sensors and its impact on society. Pursuing this central objective, we hope to have created a technically and socially 
invigorating atmosphere and our best wish is that you will enjoy your visit to the metropolitan area of Porto, Portugal, in September 2010.

\section{José Luís Santos \\ Brian Culshaw \\ José Miguel López-Higuera \\ William N. MacPherson}

